\title{
Non-aqueous retention measurements: ultrafiltration behaviour of polystyrene solutions and colloidal silver particles
}

\author{
M.A.M. Beerlage *, M.L. Heijnen, M.H.V. Mulder, C.A. Smolders, H. Strathmann \\ University of Twente, P.O. Box 217,7500 AE Enschede, Netherlands
}

Accepted 3 April 1996

\begin{abstract}
The retention behaviour of polyimide ultrafiltration membranes was investigated using dilute solutions of polystyrene in ethyl acetate as test solutions. It is shown that flow-induced deformation of the polystyrene chains highly affects the membrane retention. This coil-stretch transition is not instantaneous, but gradual. The concept of a deformation resistance has been introduced to explain this behaviour. This concept can be applied to describe the flux behaviour of the membranes during the tests as well. Solute deformation allows comparison of the pore size distributions of the membranes qualitatively. Retention measurements were also performed with silver sol particles that were prepared in mixtures of ethanol and water; these sols remain stable as long as the ethanol concentration does not exceed $57 \mathrm{vol} \%$. The sols were completely retained by the membranes, which is probably caused by the fact that the effective diameter of the particles is much larger than that observed by transmission electron microscopy.
\end{abstract}

Keywords: Membrane characterization; Non-aqueous retention measurements; Polymer flow-induced deformation; Ultrafiltration of polystyrene solutions: Ultrafiltration of silver sols

\section{Introduction}

Solute retention measurements are generally regarded as one of the most important techniques to characterize the pore size distributions of ultrafiltration membranes. The major advantage of such measurements is the fact that the characterization conditions can be very well compared to the actual ultrafiltration applications for which the membranes will be used. Complications which interfere with drying of the membranes do not occur. For polyimide membranes which are employed for non-aqueous applications, retention measurements with non-aqueous mixtures can be used as a characterization method as well.
There are still many problems involved with retention measurements, such as concentration polarization, molecular weight distribution or adsorption. Another point is the possible occurrence of flow-induced deformation of flexible polymers that are used as retention model solutes.

In this article, the retention and flux behaviour of dilute solutions of polystyrene in ethyl acetate using polyimide membranes will be described. Special attention will be focused on the flow-induced deformation of the flexible polymer chains during these retention measurements.

In addition, the retention behaviour of non-deformable silver sols will be discussed.

\footnotetext{
* Corresponding author.
} 


\section{Theoretical background and literature}

\subsection{Retention measurements}

An important industrial application for ultrafiltration membranes is found in the dairy industry, where ultrafiltration is used to concentrate whey proteins. The major problem in the ultrafiltration process is the occurrence of fouling of the membranes by the proteins [ $1-$ $3]$.

For characterization purposes, a way to avoid the specific fouling problems caused by the use of proteins, is to use synthetic polymers as model solutes in dilute solutions. Almost all characterization retention studies have been performed using aqueous systems. It is very likely that the retention and adsorption behaviour of polymers in organic solution differs from the behaviour in aqueous solutions. This will be discussed in more detail later.

The most frequently used polymers for membrane characterization are water-soluble dextrans and polyethyleneglycols (PEG), because they are commercially available in several molecular weight lengths and distributions. However, Baker and Strathmann [4] pointed out as early as 1970 , that often a discrepancy exists between the retention measurements of proteins and other polymers. They also found a retention decline for the polymer with increasing transmembrane pressure, and they ascribed this unexpected behaviour to shear-induced deformation of the flexible polymers in dilute solutions.

The literature concerning retention measurements of solutions of flexible polymers can be divided in two classes: the first group describes the retention behaviour by assuming polymer deformation, the other group shows that the behaviour of polymers can be described by assuming that the solutes are non-permeable or nondraining rigid spheres, like proteins.

In the latter case the sieving mechanism can be used, which has been described mathematically by Ferry [5]. In the literature several modifications of these equations were given to describe the sieving mechanism in the absence of fouling $[6,7]$; the values for the retention resulting from these models show little variation.

A breakthrough on the understanding of polymer deformation came in 1976, when De Gennes formulated his scaling laws for flexible polymers [8]. Daoudi and Brochard [9] used these scaling laws for a physical description of the transport of flexible polymers through cylindrical and conical pores. Nguyen and Néel $[10,11]$ showed that the flow-induced deformation as described by scaling laws applies qualitatively to their experimental results on filtration of dilute solutions of fractionated PEG and dextran through various ultrafiltration membranes. Long and Anderson found that flow-induced deformation is not only restricted to water-soluble polymers, and that deformation is not dependent on the solvent quality (which influences the molecular radius) [12,13].

Poyen et al. [ 14] showed that the non-draining rigid sphere model is only valid as long as the pore radius is larger than the solute radius, i.e., $\lambda=r_{\mathrm{s}} / r_{\mathrm{p}}<1$. Comparison of the results of several retention studies confirms this conclusion: during measurements performed at $\lambda<0.5$ no flow-induced deformation took place [ $15-$ 17], while measurements at $\lambda \geq 1$ were influenced by flow-induced deformation $[10,11,13,18]$. Long and Anderson [12] postulated that when $\lambda \geq 1$, apparently flow-induced deformation takes place, but when $0.5<\lambda<1$, then the behaviour of the polymer molecules is some sort of combination of deformation and rigid sphere sieving; the latter situation is very difficult to describe. In conclusion: if $\lambda>0.6$, flow-induced deformation plays an important role during the ultrafiltration of very dilute solutions of flexible polymers.

Most retention studies used a rather broad solute molecular weight distribution, and this polydispersity can have a large effect on the mutual partial retention coefficients. For this reason the studies mentioned cannot simply be included in a comparison of the influence of $\lambda$ on the polymer behaviour during filtration. Also the effects of adsorption (solute-membrane interactions) on the retention, hydrodynamic flow through the pores and flow-induced deformation are still far from clear.

Another very important parameter, which is often not recognized, is the choice of the solvent. Until now, most studies were done with water as solvent. Since water has very specific characteristics, the use of organic solvents will give completely different results. Water has an extreme hydrogen bonding ability and may easily form clusters. Polymers that are water-soluble also have very specific properties (e.g., negative heat of dilution, sometimes occurrence of a lower critical solution temperature (LCST), strong tendency to aggregate and adsorb). As a consequence, the normal 
Flory-Huggins theory is often not valid for these aqueous polymer solutions. Extended reviews on watersoluble polymers can be found in the literature $[19,20]$.

The major part of water-soluble polymers contain both hydrophobic and hydrophilic moieties. A solution in water may result in a structure similar to globular proteins: intramolecular hydrophobic interaction and stabilization of this structure by strong interactions between the shell of hydrophilic groups and a surrounding layer of bound water [21]. These water-specific interactions may have a very pronounced influence on the magnitude of the elastic restoring ability, and diminish the possibility to deform; the solute molecules might be regarded as rigid spheres, even at very high shear rate. For this reason a comparison of aqueous and non-aqueous retention studies will be very difficult.

Summarizing: there are several demands that need to be fulfilled for reliable retention measurements with flexible macromolecules:

- adsorption must be excluded;

- concentration polarization must be minimized;

- use of monodisperse solutes excludes mutual effects;

- solute-solvent interactions should be understood; solvent-specific interactions have to be minimized

Here, polystyrene (PS) in ethyl acetate was chosen as a model system. The expected solute to pore radius, $\lambda$, was $\geq 1$ for the majority of pores, as estimated from permporometry measurements for the tested membranes [22]; in practice, the value of $\lambda$ was always larger than 0.5 . The $\lambda$ used here is based on the ratio of the hydrodynamic solute size to the pore size. The results can also be compared to other non-aqueous retention measurements, with PS as solute. In addition, the adsorption ability of PS on the polyimide membranes has been examined.

Colloidal systems cannot deform during permeation. In the past metal sols in water have been used for membrane characterization [23,24].

In the literature there are only a few examples of non-aqueous sols, and an attempt was made here to use the silver sol system of Kim et al. [24], and replace some of the water by ethanol. In this way, the flux of the silver sol system can be related to the pure ethanol permeability and degradation of the polyimide is not very likely to occur.

\subsection{Flow-induced deformation}

A polymer chain in dilute solution can be regarded as a random coil, with a certain radius of gyration $r_{\mathrm{g}}$. The hydrodynamic radius $\left(r_{\mathrm{h}}\right)$ of a polymer is frequently approximated by the Stokes-Einstein radius of the polymer. When a large mechanical force is exerted on the polymer chain, this coil model is not valid anymore: the chain can be described better by a string of statically and dynamically independent "blobs" $[8,25]$, all with a characteristic blob size.

Consider an isolated polymer random coil ( size $r_{\mathrm{g}}$ ) in a very dilute solution at the entrance of a pore with size $r_{\mathrm{p}}$, with $r_{\mathrm{g}}$. When pressure is applied, solvent will flow through the pore. At low pressures the flow through the pore is small, and consequently the shear exerted by the frictional force on the polymer chain is low. The chain tends to return to its original, entropically favourable coil configuration by Brownian movement, due to an elastic force counteracting the frictional force.

When the transmembrane pressure increases, the flow through the pore increases and so does the frictional force. At a certain flow rate, the two forces are in equilibrium. When this critical shear $\left(S_{c}\right)$ is exceeded, the polymer chain unrolls into a chain of blobs; this process is called flow-induced deformation. The characteristic size of the polymer is now the blob size, and because this value is smaller than the pore size, the deformed polymer can enter the pore easily. A schematic representation of flow-induced deformation is given in Fig. 1.

Nguyen and Néel [11] related the critical shear to a critical flow rate through the pore, $O_{\mathrm{p}}\left[\mathrm{m}^{3} / \mathrm{s}\right]$. Daoudi and Brochard [9] assumed that the critical shear has to be reached at a distance from the pore opening that is at least of the order of the size of the dissolved polymer, $r_{\mathrm{g}}$. Consequently, $J_{\mathrm{c}}$ is the critical flow rate through the membrane $[\mathrm{m} / \mathrm{s}]$, which is then:

$J_{\mathrm{c}} \cong \frac{k_{\mathrm{B}} T \epsilon}{\eta_{\mathrm{o}} r_{\mathrm{p}}^{2}}=\frac{k_{\mathrm{B}} T n}{\eta_{\mathrm{o}}}$

Here, $k_{\mathrm{B}}$ is the Boltzmann constant $[\mathrm{J} / \mathrm{K}], T$ is the temperature $[\mathrm{K}], \eta_{\mathrm{o}}$ is the solvent viscosity [Pa s], $\epsilon$ is the surface porosity $n \pi r_{\mathrm{p}}^{2}[-], r_{\mathrm{p}}$ is the pore radius $[\mathrm{m}], n$ is the number of pores per membrane surface area $\left[/ \mathrm{m}^{2}\right]$. This means that the critical flow rate or the critical flux through the membrane is not dependent on 


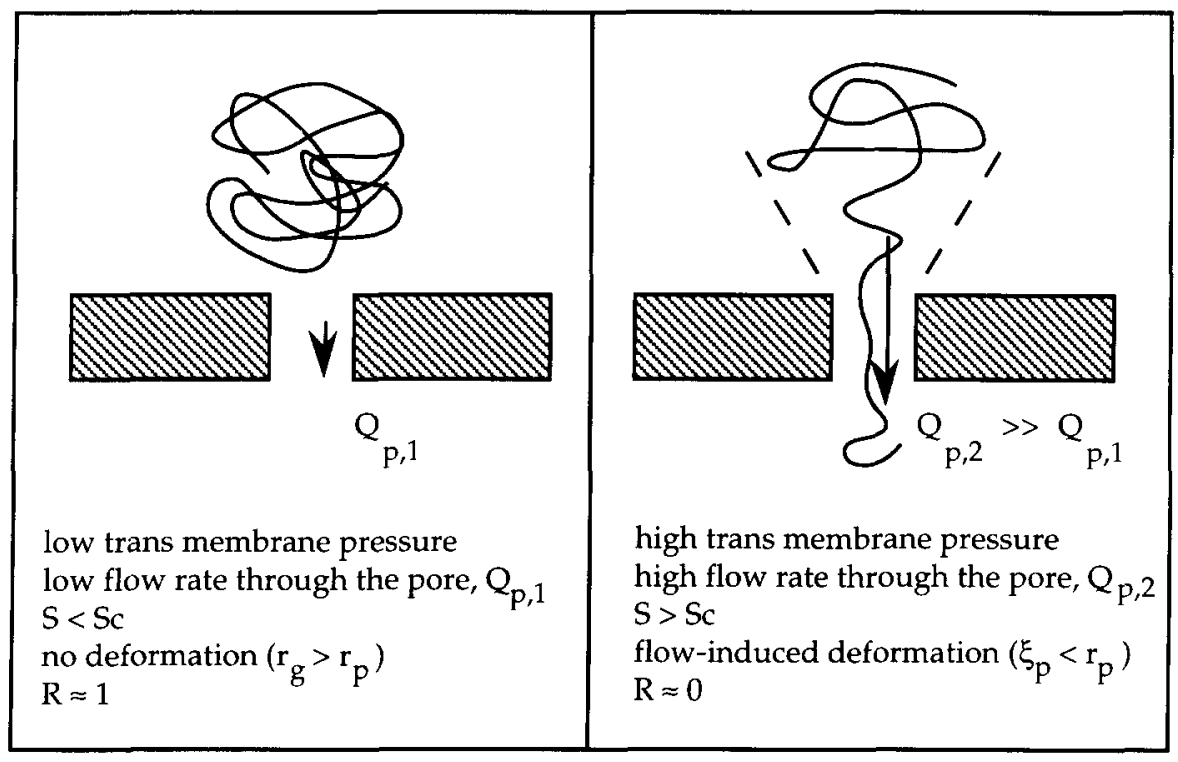

Fig. 1. Schematic drawing illustrating the flow-induced deformation.

the size of the pore, which is of course very surprising. It is also independent of the size of the original polymer coil, and of the polymer concentration (as long as the polymer solution is very dilute). One remark has to be made here: the independence of $J_{c}$ on the pore radius is mainly a consequence of the boundary condition defining the minimal distance from the pore opening, whereas this demand is only an assumption that is strongly dependent on the flow field. (For the lowporosity membranes used here, the pore entrances are situated relatively far from each other. Therefore, it is assumed that each pore has its own typical flow field and its own value of $J_{. .}$)

On the other hand, $J_{\mathrm{c}}$ is dependent on the number of pores, $n$. The independence of $J_{\mathrm{c}}$ on the pore size has been confirmed by several experimental studies $[13,15,26]$; also the independency of $J_{c}$ on the molecular weight, the radius of gyration or the hydrodynamic Stokes-Einstein radius of the solute has been proven experimentally [11-13,26-28]. The choice of solvent quality is not important as well, as may be deduced from the fact that the radius of gyration has no effect on $J_{c}$.

In the present study, more attention will be directed to the dependence of the critical flow rate through the membrane on the number of pores, because it is suggested that the comparison of the retention curves for different membranes can give more qualitative infor- mation about the number of pores. In this way, deformation studies may form an additional membrane characterization technique.

The flow-induced deformation theory as described above, is only valid for dilute solutions; this means that the polymer concentration $c$ has to be far below the overlap concentration, $c^{*}[29]$. Below $c^{*}$, the polymer coils have no interaction with each other. In the vicinity of $c^{*}$ they start to "overlap", and far above $c^{*}$ polymer chains form an entangled network. A detailed discussion on retention behaviour and flow-induced deformation at higher concentrations is beyond the scope of this article; the measurements will be restricted to dilute solutions.

Many characteristic phenomena that occur during retention measurements can be understood from scaling concepts combined with the coil-stretch transition (flow-induced deformation). However, there are a number of drawbacks. The first and most important one is the qualitative character of the scaling theory; because scaling laws do not include numerical coefficients, exact values for the critical flow rate can not be predicted. Adamski and Anderson [27] correctly mentioned that the convergent pore flow field, used by Daoudi and Brochard, probably is not right; this may introduce at least some dependence of $J_{\mathrm{c}}$ on $r_{\mathrm{g}}$. A very difficult point is the coil-stretch transition itself. De Gennes [30] reasoned that this transition should be 
very sharp; the expectation was that the retention of a porous membrane for flexible polymers would drop immediately to zero at the critical flow rate. However, experiments with track-etched mica membranes containing uniform pore sizes, showed a gradual decrease in retention [12,27]. Larson and Magda [31] reasoned that the transition is at least somewhat more gradual than predicted by De Gennes.

The coil-stretch transition by flow shear stress has recently been proven experimentally by various research groups [32-35].

In spite of these disadvantages, the deformation theory may be very helpful to explain qualitatively the typical ultrafiltration behaviour of dilute solutions of flexible polymers, in the absence of concentration polarization.

\section{Experimental}

\subsection{Materials}

Polyimide P84 (Lenzing AG, Austria) was kindly supplied by X-Flow BV. DMF, ethyl acetate and ethanol were purchased from Merck (analytical grade) and were used as received. Monodisperse polystyrene fractions were purchased via Waters from Tosoh Corp., with $M_{\mathrm{w}}=96400 \mathrm{~g} / \mathrm{mol}$ and $M_{\mathrm{w}} / M_{\mathrm{n}}=1.01$ (TS144), and $M_{\mathrm{w}}=355000 \mathrm{~g} / \mathrm{mol}$ and $M_{\mathrm{w}} / M_{\mathrm{n}}=1.02$ (TS-85).

\subsection{Membrane preparation}

PI-powder was dried at least $24 \mathrm{~h}$ at $150^{\circ} \mathrm{C}$ in vacuum. Casting solutions in DMF were prepared immediately after the drying procedure, and the air inside the erlenmeyer was replaced by nitrogen. After $24 \mathrm{~h}$ of stirring (without heating) the solutions were filtered with a Bekipor ${ }^{\mathrm{R}} 25 \mu \mathrm{m}$ stainless steel filter from Bekaert Corp. The solutions were degassed overnight.

A film of $0.20 \mathrm{~mm}$ thickness was cast on a dry and clean glass plate and immediately immersed in the coagulation bath, containing ethanol or demineralized water. The residence time in the bath was at least 10 min, but the membranes precipitated immediately. The prepared membranes were flushed for $24 \mathrm{~h}$ with water to remove DMF; the water was replaced by ethanol, in which the membranes were stored.
Before the membranes were applied for filtration of ethyl acetate or polymer solutions in ethyl acetate, they were pre-conditioned in this liquid for at least two days.

\subsection{Polymer feed solutions}

Polystyrene samples were dissolved in ethyl acetate at various feed solution concentrations, $c_{0}$, to a maximum of $1 \mathrm{~g} / 1$ (for the high $M_{\mathrm{w}}$ a feed concentration of $0.7 \mathrm{~g} / 1$ was used).

To determine hydrodynamic radii for PS 96400 and PS 355000 , the bulk diffusion coefficients in dilute solution have to be known. Some results, obtained by quasi elastic light scattering, are presented in the literature for PS in dilute solutions in ethyl acetate at $20^{\circ} \mathrm{C}$ [36,37]. Analogously to Kathawalla and Anderson [38], these data were fitted by a power law dependent on the molecular weight with an additional temperature and viscosity correction: $D_{0} \sim 3.296 \times$ $10^{-8} \times M_{\mathrm{w}}^{-0.533}$.. Hydrodynamic radii, $r_{\mathrm{h}}$, can then be calculated using the Stokes-Einstein relation. The radius of gyration, $r_{\mathrm{g}}$, can be estimated using a relation between $r_{\mathrm{g}}$ and $r_{\mathrm{h}}$ for PS in good solvents [38]:

$\frac{r_{\mathrm{g}}}{r_{\mathrm{h}}}=1.48 \pm 0.03$

Guillot et al. [36] showed that ethyl acetate is a good solvent for PS.

The literature concerning the value of the overlap concentration is quite indistinct, several relations were applied to calculate $c^{*}$. Because the purpose of the present work is to investigate ultrafiltration behaviour at concentrations far below $c^{*}$, two examples of calculations are given here. Eq. (3) shows the overlap concentration according to Doi and Edwards [39], while Eq. (4) gives the overlap concentration according to Des Cloizeaux and Jannink [40]:

$c^{*}=\frac{M_{\mathrm{w}}}{\frac{4}{3}} \pi r_{\mathrm{g}}^{3} N_{\mathrm{A}}$

$c^{*}=\frac{M_{\mathrm{w}}}{\left(\sqrt{2} r_{\mathrm{g}}\right)^{3} N_{\mathrm{A}}}$

where $N_{\mathrm{A}}$ is Avogadro's number.

The characteristics of the polystyrenes used in this study are summarized in Table 1 . The radius of gyra- 
Table 1

Characteristics of the monodisperse polystyrene samples used

\begin{tabular}{ccccc}
\hline PS mol. weight $(\mathrm{g} / \mathrm{mol})$ & $r_{\mathrm{h}}(\mathrm{nm})$ & $r_{\mathrm{g}}(\mathrm{nm})$ & $c^{* \mathrm{a}}[\mathrm{g} / 1]$ & $c^{* \mathrm{~b}}[\mathrm{~g} / \mathrm{l}]$ \\
\hline 96400 & 7.07 & 10.5 & 33 & 49 \\
355000 & 14.1 & 20.9 & 15 & 23 \\
\hline
\end{tabular}

"From Eq. (3).

${ }^{b}$ From Eq. (4).

tion has been estimated from Eq. (2). From Table 1 it can be concluded that the feed concentrations used were far below any value of $c^{*}$, even far below the value of $1 / 8 c^{*}$, which was advised by Bishopet al. [41]. Teraoka et al. [42] recently showed by measurements of diffusion of PS in porous glasses, that the concentration regime inside the pore is definitely dilute, if the ratio of $c_{0} / c^{*} \leq 0.2$ and $c^{*}$ has been defined according to Eq. (4). The feed concentrations are also far below this latter value.

Permporometry measurements [22] showed that for the membranes used the major part of the pore radii is smaller than $7 \mathrm{~nm}$, so here $\lambda=r_{\mathrm{s}} / r_{\mathrm{p}} \geq 1$. In addition, for all pores $\lambda>0.5$. It should be realized that these pore size distributions were determined for membranes in a dry state, which may result in a change in morphology.

Concentrations of PS-solutions were determined with a Philips PU 8720 UV/Vis scanning spectrophotometer at a wavelength of $260 \mathrm{~nm}$.

\subsection{Silver sols}

Silver sols were prepared according to the method described by Creighton et al. [43]: $100 \mathrm{ml}$ of $10^{-3} \mathrm{M}$ aqueous $\mathrm{AgNO}_{3}$-solution was added to $300 \mathrm{ml}$ of a $2 \times 10^{-3} \mathrm{M}$ aqueous $\mathrm{NaBH}_{4}$ solution at $0^{\circ} \mathrm{C}$ under vigorous stirring. This mixture was slowly diluted with water to a concentration of $0.1 \times 10^{-3} \mathrm{M}$ or $0.11 \times 10^{-3} \mathrm{~g} \mathrm{Ag} / \mathrm{l}$, according to Kim et al. [24]. The resulting sol was light yellow and stable for several months, without any precipitate. The stock solution was very slowly diluted with the desired ratio of ethanol to water. Three different final concentrations were used: $0 / 100 \mathrm{v} / \mathrm{v}, 30 / 70 \mathrm{v} / \mathrm{v}$, and $57 / 43 \mathrm{v} / \mathrm{v}$ ethanol/water, respectively; these sols remained stable for at least three weeks without any visual changes. An increase of the ethanol concentration above $57 \%$ resulted in an immediate colour change to darkbrown or grey, with finally a grey precipitate. The prepared sols were used for retention measurements immediately after preparation. Concentrations of the sols were determined using a Philips PU $8720 \mathrm{UV} /$ Vis scanning spectrophotometer at $\lambda=393 \mathrm{~nm}$.

Size distributions of the sols were determined using a Jeol $200 \mathrm{CX}$ high resolution transmission electron microscope. For this purpose, drops of the sols were placed on a sample holder, after which the solvent was evaporated.

\subsection{Retention measurements}

The pure solvent permeability of the membrane for ethyl acetate or ethanol was determined in a dead-end filtration set-up ( $3 \mathrm{~h}$ filtration at $1 \mathrm{bar}$ ), prior to the actual retention measurement. Directly after this procedure, the supply tank was emptied and the pure solvent in the $400 \mathrm{ml}$ cell was replaced by the solution and stirred immediately at $1200 \mathrm{rpm}$. After adjusting the pressure the permeate samples were collected and analysed.

The retention was calculated as $R=\left(\left(c_{\mathrm{o}}-c_{\mathrm{p}}\right) / c_{\mathrm{o}}\right)$; the increase in the feed concentration, $c_{0}$, caused by retention, was always much lower than $10 \%$. For new retention measurements, clean and fresh membranes were used.

\section{Results and discussion of retention measurements obtained with polystyrene solutions}

\subsection{Influence of polystyrene molecular weight}

For the measurements only one type of membrane was used. The membranes were prepared from a 20 $\mathrm{wt} \%$ casting solution of polyimide in DMF and coagulated in ethanol. Retention values of these membranes for a solution of PS with a $M_{\mathrm{w}}$ of $96400 \mathrm{~g} / \mathrm{mol}$ and a solution of PS with a $M_{\mathrm{w}}$ of $355000 \mathrm{~g} / \mathrm{mol}$ were determined. The concentrations of the PS-solutions were 1 $\mathrm{g} / 1$ for PS 96400 and $0.7 \mathrm{~g} / 1$ for PS 355000 . The retentions were measured at two different transmembrane pressures and were stable for at least $30 \mathrm{~min}$. The results are listed in Table 2 .

The results from Table 2 can be explained by the flow-induced deformation theory. An increase in permeate flow causes a decrease in retention, which 
Table 2

Retentions of a membrane prepared from a casting solution of 20 wt.\% PI in DMF and coagulated in ethanol, for PS-solutions of two different monodisperse PS molecular weights

\begin{tabular}{lll}
\hline Pressure (bar) & PS 96 400 (g/mol) $R$ \\
$(\%)$ & $\begin{array}{l}\text { PS 355 000 }(\mathrm{g} / \mathrm{mol}) R \\
(\%)\end{array}$ \\
\hline 0.1 & 90 & 92 \\
1.0 & 0 & 0 \\
\hline
\end{tabular}

implies that the polystyrene chains can change their conformation to pass through small pores at higher permeate flow velocities.

It is obvious that the retention behaviour of these membranes is similar for the two PS molecular weights. This means that the critical flow rate through the membrane is independent of the size of the polymer coil $\left(M_{\mathrm{w}}\right)$, which conforms with Eq. (1). This result also agrees very well with the retention results that were described in literature (see theory).

The solvent flux [ $\mathrm{g} / \mathrm{min}$ ] for the 96400 -solution was almost equal to the pure ethyl acetate flux, which means that concentration polarization is negligible. Therefore, pore plugging can not be the cause of such a drastic decrease in retention. On the other hand, the flux for the 355000 -solution was lower than the pure solvent flux. This will be discussed further in the next section.

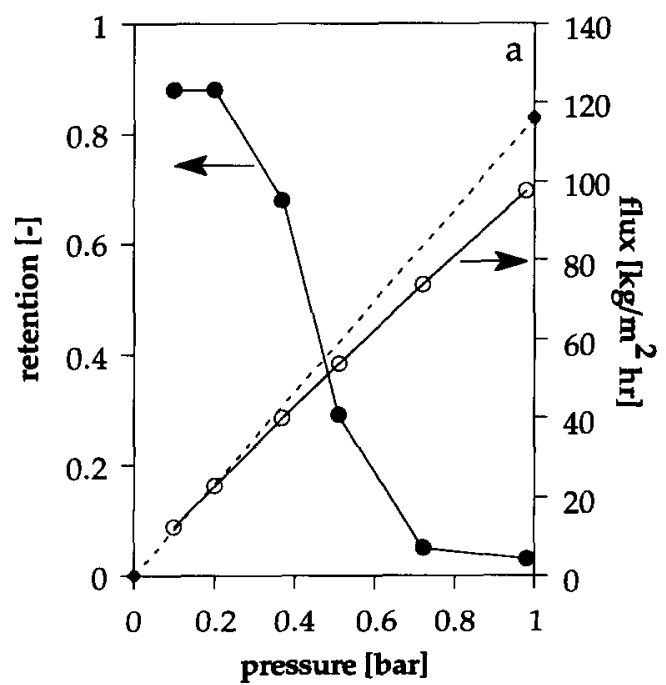

For this membrane, the pore size distribution (determined by permporometry) showed that there are some pores present that have a radius of $14 \mathrm{~nm}$, which explains that the retention is not complete for neither of these two PS solutes. Hindered diffusion experiments with dilute polystyrene solutions in ethyl acetate have been performed for the same membrane as well [44]. For both PS 96400 and PS 355000 the diffusive permeability was slightly larger than zero, which means that also according to this characterization technique there are some pores present with a radius of about 14 $\mathrm{nm}$.

\subsection{Comparison of retention curves for different membranes}

Standard retention curves were measured for six different types of membrane. Three different casting solution polyimide concentrations (18, 20 and $25 \mathrm{wt}$.\% PI in DMF) and two different coagulation baths, ethanol or water, were used. The permeabilities of these membranes for pure ethyl acetate vary roughly from 130 $\mathrm{kg} / \mathrm{m}^{2} \mathrm{~h}$ bar for the "tight" membranes (25 wt.\% PI), to about $1000 \mathrm{~kg} / \mathrm{m}^{2} \mathrm{~h}$ bar for the more "open" membranes ( $18 \mathrm{wt} . \% \mathrm{PI}$ ).

Retention curves for these membranes were determined using feed solutions of $1 \mathrm{~g} / 1$ PS 96400 in ethyl acetate; the results for the different membranes are

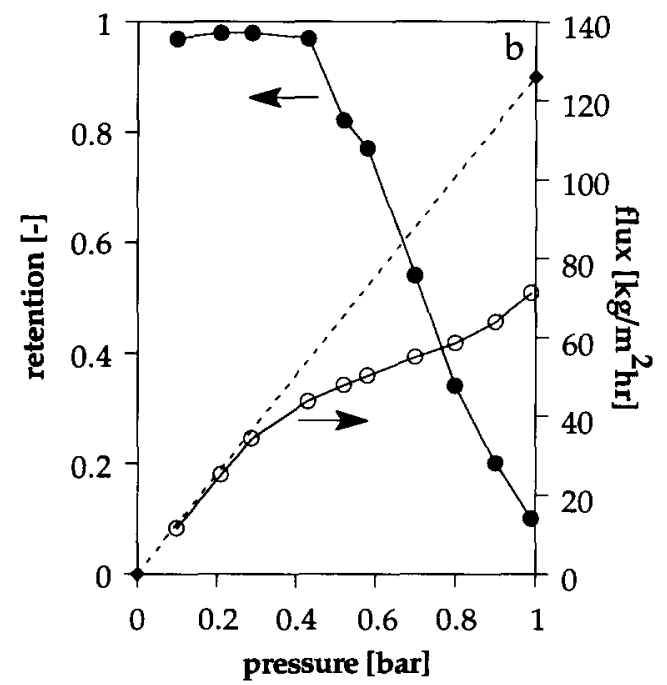

Fig. 2. Flux and retention behaviour of membranes prepared from $25 \mathrm{wt} \%$ PI in DMF and coagulated in ethanol (a) or water (b), for $1 \mathrm{~g} / 1 \mathrm{PS}$ ( 96400 ) solution in ethyl acetate. Pure ethyl acetate fluxes are represented by dashed lines. 

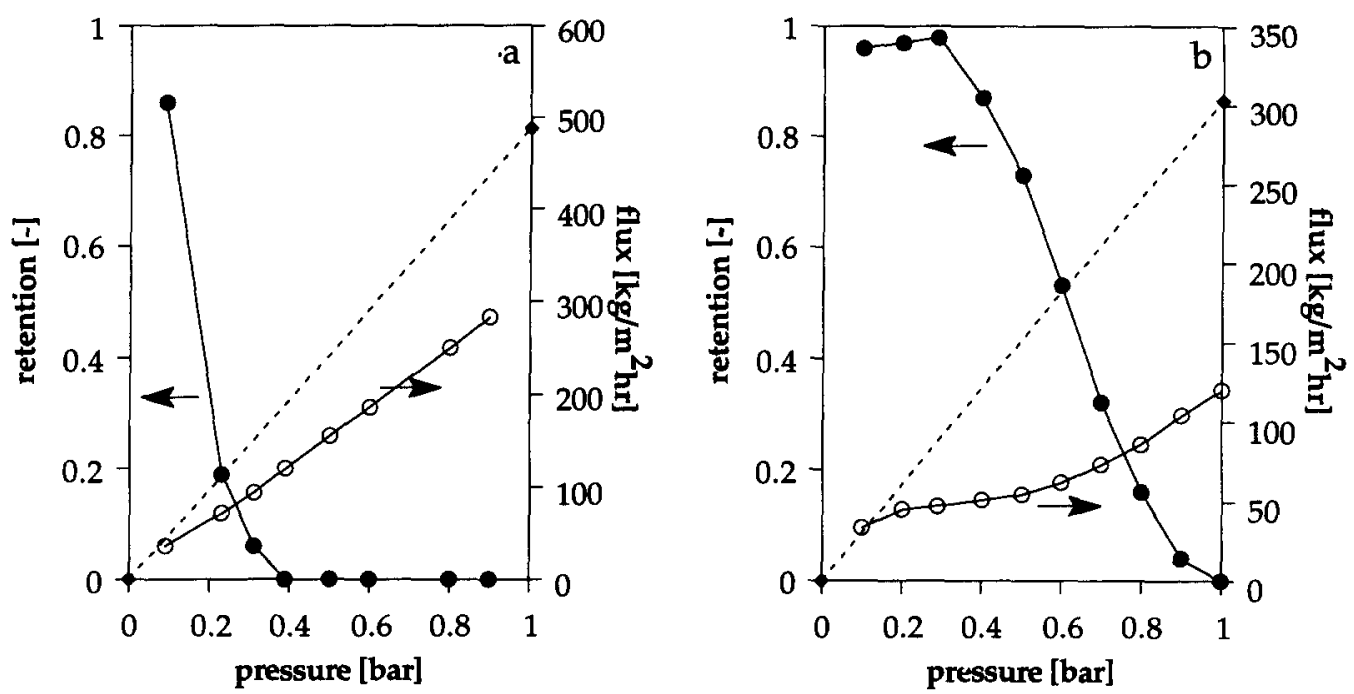

Fig. 3. Flux and retention behaviour of membranes prepared from $20 \mathrm{wt} \%$ PI in DMF and coagulated in ethanol (a) or water (b), for $1 \mathrm{~g} / 1 \mathrm{PS}$ (96 400) solution in ethyl acetate. Pure ethyl acetate fluxes are represented by dashed lines.

given in Fig. 2 (25 wt.\% PI), Fig. 3 (20 wt.\% PI) and Fig. 4 ( 18 wt.\% PI).

The measurements were very reproducible and different membranes from the same batch resulted in only minor differences in retention behaviour. A stepwise decrease in pressure gave the same results as a stepwise increase. When the same membrane was used twice for similar measurements, changes in the retention curves were negligible.

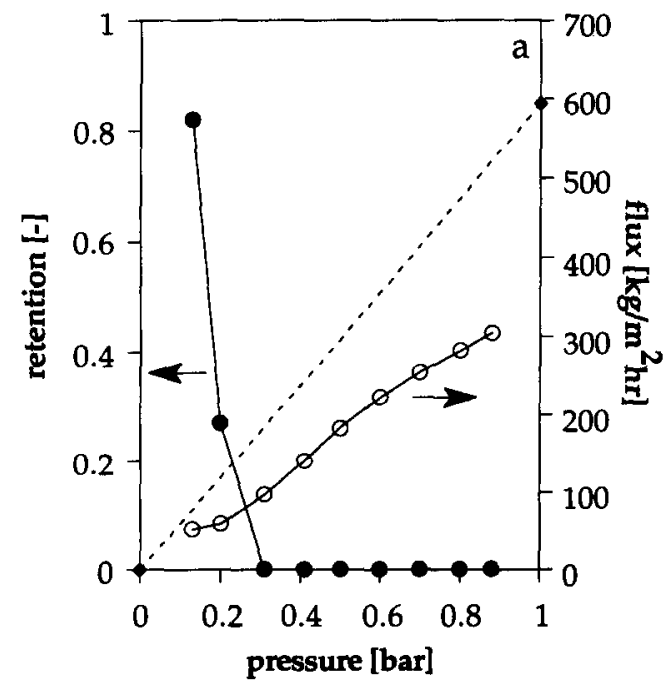

The form of the retention curves agrees well with literature data and can be explained by flow-induced deformation. For the situations that are given in Fig. 2a, Fig. 2b, and Fig. 3b, at very low pressures $(P \approx 0.1$ bar $)$ almost no deformation takes place and the retention is high and almost constant. When the pressure increases, the critical permeate flow is reached, flow-induced deformation takes place and the retention drops to zero; this happens at different pres-

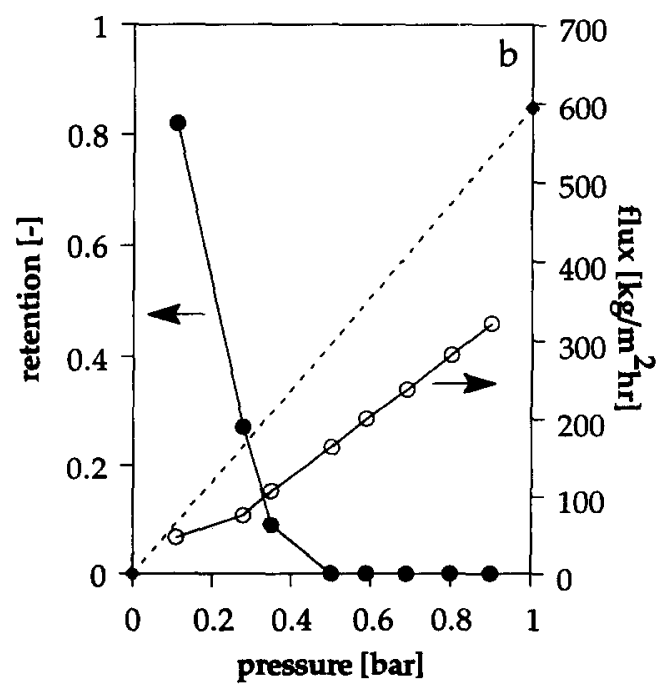

Fig. 4. Flux and retention behaviour of membranes prepared from $18 \mathrm{wt} \%$ PI in DMF and coagulated in ethanol (a) or water (b), for $1 \mathrm{~g} / \mathrm{l}$ PS ( 96 400) solution in ethyl acetate. Pure ethyl acetate fluxes are represented by dashed lines. 

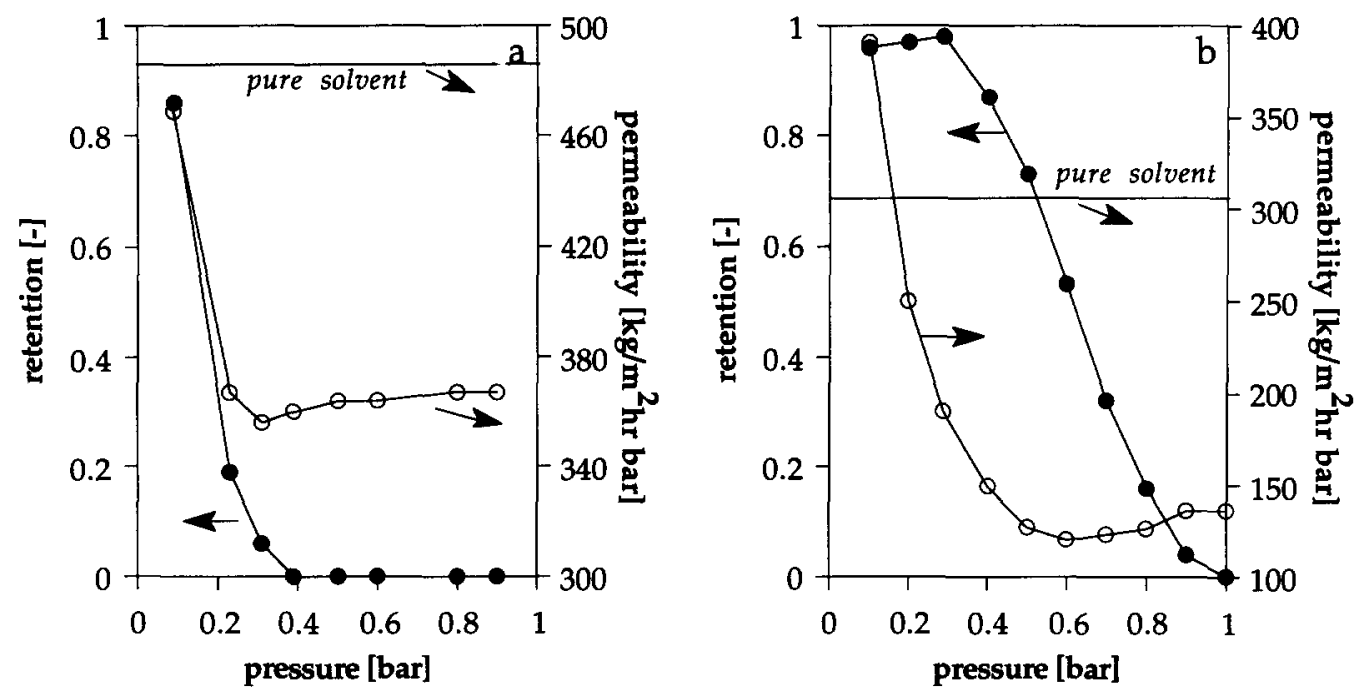

Fig. 5. Retention and solution permeability of membranes prepared from $20 \mathrm{wt}$ \% PI in DMF and coagulated in ethanol (a) or water (b), for 1 g/I PS/ethyl acetate. The flux results of Fig. 3 are normalized to the transmembrane pressure difference, units: $\mathrm{kg} / \mathrm{m}^{2} \mathrm{~h}$ bar.

sures for the different types of membranes. For the situations presented in Fig. 3a, Fig. 4a, and Fig. 4b, deformation already occurs at 0.1 bar pressure. It is obvious for all the measurements that the retention decreases with increasing pressure, which is typical for flow-induced deformation.

The flux of the solution is also given in the figures. Note that the flux is given in $\mathrm{kg} / \mathrm{m}^{2} \mathrm{~h}$ units, and is not normalized to the pressure. When the retention is high, i.e., before deformation starts, the flux is equal to the pure ethyl acetate permeability as shown in the figures by a dashed straight line. Despite the high retention, concentration polarization is negligible. As soon as deformation starts the flux deviates from linear behaviour, but still increases with pressure. When zero retention is reached the flux increases linearly with the pressure again, at a somewhat lower level than the pure solvent flux. This can be made more clear in Fig. 5, where the results of Fig. 3 are replotted, using a pressure normalized flux (i.e., the solution permeability).

It is clear that the strong decline in retention by no means can be explained by concentration polarization, because in all cases described in the literature where concentration polarization occurs, the permeability finally is decreasing with pressure.

As already mentioned before, it is quite plausible that the coil-stretch transition occurring during flowinduced deformation, is not as sharp as De Gennes predicted [30] but is more gradual, as stated theoretically [31] and experimentally $[11,12,27]$. The frictional energy, necessary to deform a polymer molecule, is supplied by the kinetic energy of the convective permeate flow. A gradual coil-stretch transition in this respect corresponds with a gradual decrease in kinetic energy of the permeate flow. This decrease results in an additional resistance to permeate flow, a sort of "deformational resistance", that becomes constant when all the polymer molecules are deformed at a retention of zero (see Fig. 5). At this point, the flux is again linearly dependent on the pressure, at a somewhat lower level than the pure ethyl acetate permeability line (as in Fig. 5). However, part of the loss in kinetic energy of the permeate flow can also be ascribed to the friction of the solvent molecules around the deformed chain with the pore wall (especially for very small pores, with a pore size that is of the order of the blob size). Nevertheless, it is suggested here that this wall drag resistance that is mainly present in very small pores is only a fraction of the decrease in kinetic energy, that is indicated by the decrease in permeability.

Summarizing, a new resistance model for ultrafiltration of a very dilute solution of flexible polymers (retention measurements for membrane characterization) is suggested (see Fig. 6).

With respect to the deformational resistance, one may conclude from Fig. 2, Fig. 3 and Fig. 4 that in 


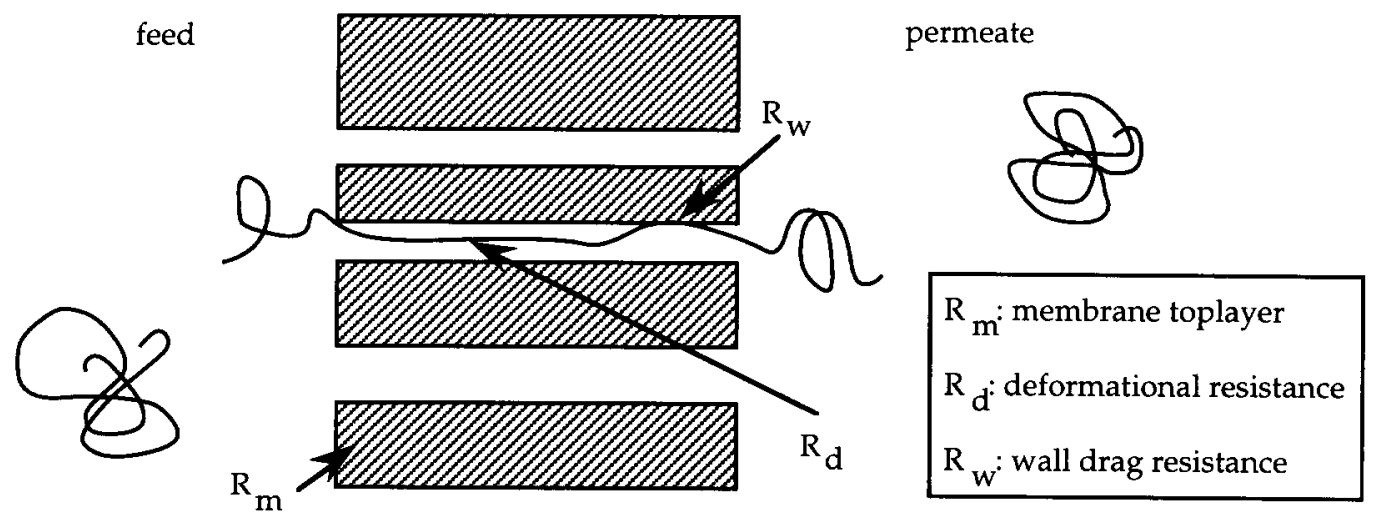

Fig. 6. Schematic drawing depicting the hydrodynamic resistances occurring during ultrafiltration of very dilute solutions of flexible polymers.

most cases the resistance is higher for membranes coagulated in water, than for membranes coagulated in ethanol. A possible explanation could be that the pores in water-coagulated membranes are longer (thicker toplayer) or more tortuous, so that the deformation that has to be maintained for a longer time requires more energy than in the case of ethanol-coagulated membranes. This hypothesis is very difficult to prove experimentally. There is no other characterization method available to determine the tortuosity of the pores in the toplayer, while for the determination of the skin thickness until now only an aqueous gold-sol filtration method was used [23].

It is also possible that the flow-induced deformation of a polymer with a higher molecular weight causes a higher deformational resistance than a polymer with a lower molecular weight (see results in former section). If this is true, then there is no influence on the form of the retention curve, but the flux at zero retention will be lower for the higher molecular weight polymer.

The critical permeate flow for the six membranes is reached at very different pressures, but of course, the pure ethyl acetate permeability of the membranes also shows a large variation. The retention curves of the six types of membranes from Fig. 2, Fig. 3 and Fig. 4 are plotted in Fig. 7 versus the flux through the membrane. The retention curves of the membranes coagulated in ethanol are situated more to the left hand side compared to the membranes coagulated in water. Because the critical flow rate through one pore, $Q_{\mathrm{p}, \mathrm{c}}$, is equal for both cases (independent of pore size), this difference in critical flow rate through the membrane, $J_{c}$, can only result from a difference in the number of pores, $n$, as predicted by Eq. (1).
This means that a membrane coagulated in water has more pores than a similar membrane coagulated in ethanol $\left(n_{\text {water }}>n_{\text {ethanol }}\right)$. Also a trend for the polymer concentration can be observed: membranes from 18 wt.\%-solutions have more pores than membranes from 20 or $25 \mathrm{wt} . \%$-solutions: for these higher concentrations parts of the curves overlap, so $n_{20} \approx n_{25}$. Combining this latter result with the fact that the pure solvent permeability for $20 \mathrm{wt}$.\% PI-membranes is about a factor five higher than for $25 \mathrm{wt}$.\% PI-membranes, one may conclude that 20 wt.\% PI-membranes contain larger pores than $25 \mathrm{wt} . \%$ PI-membranes. This result for wet membranes agrees with the results of permporometry for comparable membranes in the dry state [22]. (It is also possible that the length of the pores for $25 \mathrm{wt} . \% \mathrm{PI}$-membranes is larger than for the 20 wt. \% PI-membranes, but there are no experimental data available for the toplayer thickness.)

To our knowledge, this is the first time that flowinduced polymer deformation studies are used for membrane characterization purposes. Of course, this type of characterization is only qualitative, but it may be possible to compare pore size distributions of different membranes made of the same material; this last demand is necessary to completely exclude the influence of interactions between solute polymer and membrane material as a complicating factor.

This method may be useful in relation to membrane formation.

\subsection{Influence of feed concentration}

Retention curves were determined at a lower feed concentration for two types of membranes. For $25 \mathrm{wt} . \%$ 


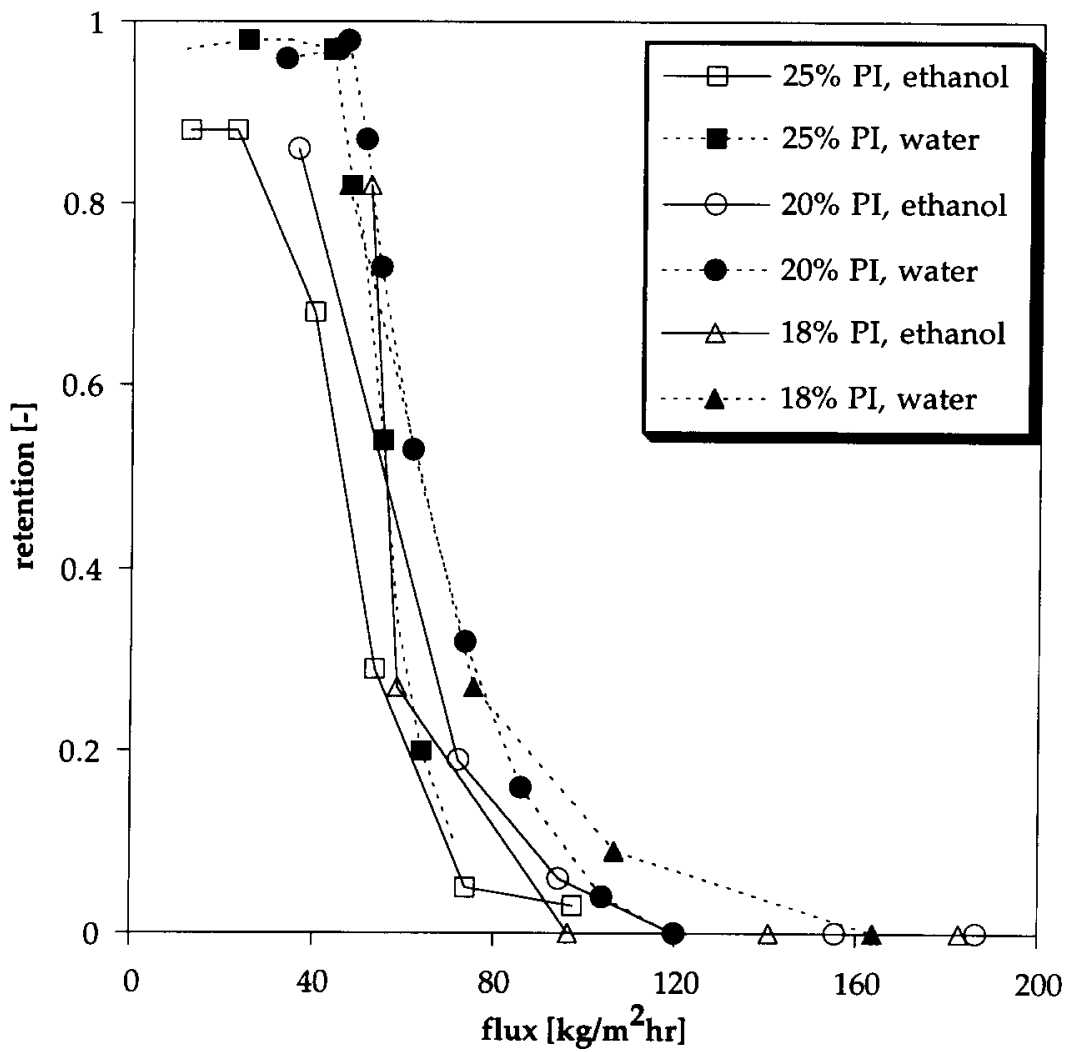

Fig. 7. Retention for $1 \mathrm{~g} / \mathrm{l}$ solutions of PS (96 400) in ethyl acetate as a function of the flux through the membrane; comparison of six different types of membranes.

PI-membranes coagulated in ethanol a feed concentration of $0.34 \mathrm{~g} / \mathrm{I}$ was used, while for $25 \mathrm{wt} . \%$ PI-membranes coagulated in water a feed concentration of 0.58 $\mathrm{g} / \mathrm{l}$ was tested. The results are given in Fig. 8 .

The results in Fig. 2a and Fig. 8a look very similar, even if one takes into account that two different membranes (from the same batch) have been measured. For Fig. $2 b$ and Fig. 8 b, the retention curves are almost similar, but the flux curves seem to deviate a little from each other.

This means that the feed concentration has no influence on the retention curve, which agrees well with the flow-induced deformation theory. This is an experimental proof for Eq. (1), from which it has already been concluded theoretically that the critical flow rate is independent of the (dilute) polymer concentration.

On the other hand, the feed concentration seems to have an influence on the flux behaviour for some of the membranes. As already mentioned, the deformation theory and almost all published experimental results involve the retention behaviour; however, the dependence of the flux was never described satisfactorily. The flux behaviour described here can also be explained by the higher resistance to deformation in the case of water-coagulated membranes. An increase in feed concentration simply means that there are more polymer molecules that have to be deformed and that the friction with the pore wall increases, thus the energy loss is larger. The increased energy loss causes an increase in resistance to permeate flow, so a larger deviation from the pure ethyl acetate flux has been observed, while the same retention curve was maintained.

\subsection{Adsorption tests}

The pure ethyl acetate permeability of the membrane after the retention measurement was a little lower than before, about $5 \%$. 

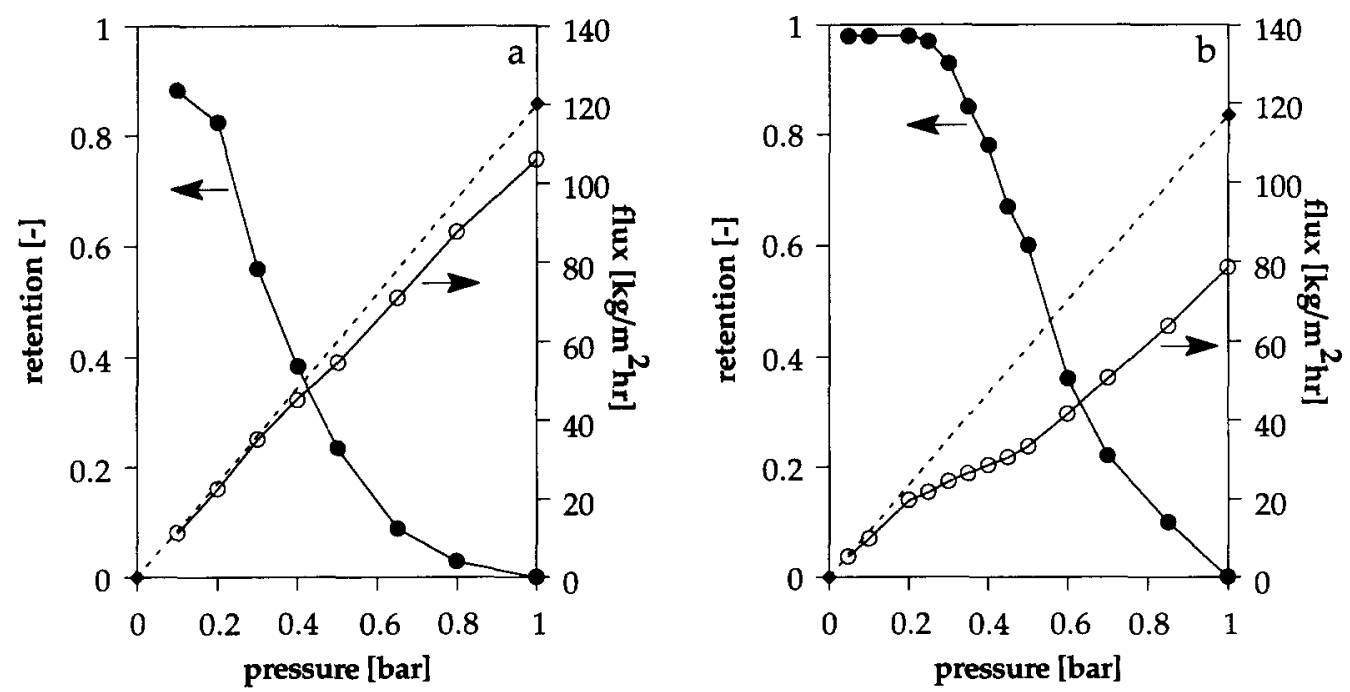

Fig. 8. Influence of feed concentration on flux and retention behaviour of a membrane prepared from 25 wt.\% PI in DMF and coagulated in ethanol for $0.34 \mathrm{~g} / \mathrm{l}$ PS solution (a), and of a membrane prepared from $25 \mathrm{wt} \% \mathrm{PI}$ in DMF and coagulated in water for $0.58 \mathrm{~g} / 1 \mathrm{PS}$ solution (b).

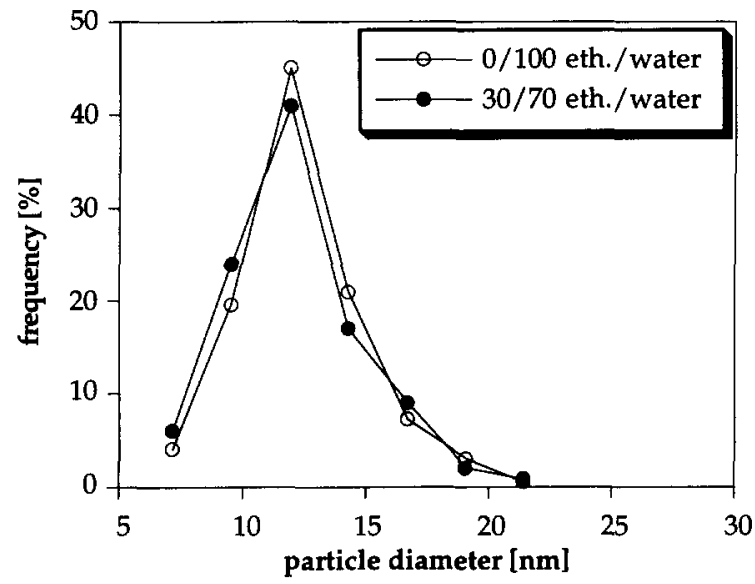

Fig. 9. Particle diameter distributions (determined by TEM) for silver sols diluted with water or water/ethanol.

To investigate whether this decrease is caused by fouling, membranes were put in a PS 96400 solution in ethyl acetate for $24 \mathrm{~h}$, while similar membranes were put in pure ethyl acetate. The ethyl acetate permeability after this treatment showed about $5 \%$ decrease for both tests. This means that the small decrease in permeability after retention measurements is not caused by pore plugging nor by adsorption at the membrane and pore surface, but just by immersion in ethyl acetate. Only after seven days in ethyl acetate, was a constant ethyl acetate permeability observed. Probably the pre-con- ditioning time in ethyl acetate of two days, as is used for all measurements, is not long enough. Ethyl acetate is the only solvent that showed this behaviour; preconditioning times of two days in hexane, toluene or acetone always gave constant permeabilities. For the ethyl acetate measurements the same pre-conditioning times were always used, i.e., it may be assumed that small differences are negligible or, at least, comparable for all membranes. An important conclusion is that polystyrene does not adsorb on polyimide membranes, as was expected.

\section{Retention measurements obtained with silver sols}

\subsection{Stability and particle diameters of silver sols}

Several attempts were made to obtain silver sols in mixtures of ethanol and water. Experimentally, it has been found necessary to make a standard stock solution based on water only. The presence of only a few percent of ethanol resulted almost immediately in a grey mixture with visible particles. Dilution mixtures containing ethanol have to be added very slowly and dropwise, during vigorously stirring. All stable light-yellow solutions showed an absorption peak at $\lambda=393 \mathrm{~nm}$, which 
agrees with the results of Kim et al. [24]. As soon as the colour of the solution changed, this peak disappeared. This seems to be a very good indication of the stability of the sol.

The maximum ethanol content of the final mixture was found to be $57 \%$; this mixture still had a yellow colour, which was a little bit darker than with $30 \%$ ethanol or $100 \%$ water, and the sol was stable for at least two weeks. The mixture with $30 \%$ ethanol had exactly the same appearance as the $100 \%$ water sol. Comparison of numerous TEM-photographs showed, that the sols containing $0 \%$ ethanol or $30 \%$ ethanol look very much the same: they have a similar size distribution and they are well separated. For the sol containing the largest amount of ethanol, the particles start to form aggregates; this sol obviously is close to the stability treshold. Particle diameter distributions for the first two sols are shown in Fig. 9. The curves for both sols show a maximum at a particle diameter of $12 \mathrm{~nm}$; both distributions are slightly broader than the distribution determined by Kim et al.

\subsection{Retention measurements with silver sols}

The freshly prepared sols were used for retention measurements. All the results showed almost complete retention, even when more open membranes were used, like the $18 \mathrm{wt}$ \%-polyimide membranes. The flux of the diluted sol was about the same as the pure ethanol permeability for the sols of $30 / 70$ and $0 / 100$ ethanol to water ratio; after the retention measurements the pure ethanol permeability was equal to the permeability before the retention measurements. This corresponds quite well to the results of Kim et al. [24], who found complete retention and the formation of an "open" cake layer on top of the membrane surface for PM 30 ultrafiltration membranes. This cake layer is formed by flocculation of the colloids, which means that the size of the particles becomes much larger than the original colloid size.

When the $57 / 43$ ethanol to water ratio sol was used, the flux of the sol and the ethanol permeability after the retention measurement were lower than the pure ethanol permeability before the retention measurement. It is very likely that these sol particles become unstable during the retention measurement, so that they fall apart into smaller components. The smaller molecules may enter the pores and thereby cause pore plugging and an irreversible flux decline.

\section{Comparison of retention measurements of polystyrene and silver sol solutions}

Silver sols are undeformable particles. In addition, polystyrene chains remain randomly coiled at very low permeate flows, e.g., at transmembrane pressures slightly larger than zero. The radius of gyration for PS 96400 in ethyl acetate is about $7.1 \mathrm{~nm}$, while the particle radius for the silver sols (determined by TEM) varies from 4 to $10 \mathrm{~nm}$ with a maximum at $6 \mathrm{~nm}$. Despite the smaller size of the majority of the silver sols, the retention of all membranes tested was almost 1. It is very likely that the specific properties of silver sols are the cause of the complete rejection. The stability of these sols is assured by the presence of a large quantity of water, which is already an indication of the importance of charges. It is possible that the double layer thickness, as given by the Debye length $\kappa^{-1}$, around the sol particles increases the effective size of the silver sols. Kim et al. [24] determined for these silver sols a Debye length of 5-8 nm, which means that the effective particle radius during retention measurements can be twice as large as predicted by TEM. In addition, flocculation increases the particle size drastically.

Pore size distributions determined by permporometry showed that especially for the more open membranes (i.e., $18 \mathrm{wt} . \% \mathrm{PI}$ ) a reasonable number of pores with radii between 6 and $10 \mathrm{~nm}$ and even some larger pores were present in the dry membrane. The fact that also for $18 \mathrm{wt} \%$ PI-membranes the retention is almost 1 , means that the effective sol particle radius should be larger than these dry state pore radii. It might be possible that in a wet state the pore size distribution is shifted to higher pore radii; then the silver sols are even larger than these wet state pore radii. Unfortunately, no information could be obtained about this wet state pore size distribution.

Anyway, the conclusion can be drawn that the major part of the pores, for all the tested polyimide ultrafiltration membranes, will have radii smaller than $7 \mathrm{~nm}$; based on the high retentions for PS 96400 at very low pressures; this conclusion agrees well with the dry-state pore size distributions obtained by permporometry. 


\section{Conclusions}

The retention behaviour of very dilute solutions of polystyrene in ethyl acetate through phase-inversion polyimide membranes could be described by the occurrence of flow-induced deformation. The concept of a deformational resistance was introduced to explain the step-like flux decline, in the absence of concentration polarization.

Flow-induced deformation studies for non-aqueous systems were carried out to compare qualitatively the pore size distributions of six types of PI-membranes. It was found that the number of pores per unit area, $n$, for membranes coagulated in water is larger than that for similar membranes coagulated in ethanol. In addition, the deformational resistance is larger in the case of membranes coagulated in water, compared to membranes coagulated in ethanol, which may indicate differences in pore length or tortuosity.

The number of pores in $20 \mathrm{wt} . \%$ PI-membranes was about equal to the number of pores in $25 \mathrm{wt} . \% \mathrm{PI}-$ membranes, so the large pure solvent permeability difference between these types has to be explained by the presence of relatively larger pores in the $20 \mathrm{wt} . \%$ PImembrane.

Flexible polymer deformation studies were shown to be useful to relate qualitatively membrane formation parameters to pore size distributions of the final membranes. Deformation characterization is a wet-state technique, which is an advantage over the dry-state permporometry.

It is obvious from the results that it is not possible to use this kind of retention measurements, i.e., dilute nonaqueous solutions of flexible polymers, as quantitative characterization technique to calculate membrane pore size distributions. Furthermore, it is suggested here to be very careful in interpreting aqueous retention measurements, since water as a solvent may have a large influence on the results, which is often not recognized.

Silver sols were prepared in different mixtures of ethanol and water, to obtain small non-deformable solutes. It was found that when the final ethanol concentration exceeds $57 \%$, the sol particles start to form aggregates. A large quantity of water is necessary to ensure the stabilization of the sol particles. The presence of a double layer around the sol particles does increase the effective hydrodynamic diameter, i.e., the effective diameter is larger than the mean particle diam- eter of $12 \mathrm{~nm}$, as determined by transmission electron microscopy. The retention for stable silver sols in ethanol/water mixtures or water was almost complete for all membranes. Comparison of this result with the retentions for PS at very low pressures, i.e., molecule deformation may be neglected and the radius of gyration is $7 \mathrm{~nm}$, also supports the presence of a much larger effective diameter of the silver sols.

\section{Acknowledgements}

The authors would like to thank Mr. S. Visser for performing part of the experiments.

\section{References}

[1] A.G. Fane, Ultrafiltration: factors influencing flux and rejection (Ch. 4), in R.J. Wakeman (Ed.), Progress in Filtration and Separation, Elsevier, Amsterdam, 1986, p. 101.

[2] M. Cheryan, Modelling of ultrafiltration processes (Ch. 4), Fouling of ultrafiltration membranes (Ch. 6), Ultrafiltration Handbook, Technomic, Lancaster, 1986, p. 73 and 171.

[3] G.B. van den Berg and C.A. Smolders, Flux decline in ultrafiltration processes, Desalination, 77 (1990) 101.

[4] R.W. Baker and H. Strathmann, Ultrafiltration of macromolecular solutions with high-flux membranes, J. Appl. Polym. Sci., 14 (1970) 1197.

[5] J.D. Ferry, Ultrafilter membranes and ultrafiltration, Chem. Rev., 18(3) (1936) 373.

[6] E.A. Mason, R.P. Wendt and E.H. Bresler, Similarity relations (dimensional analysis) for membrane transport, J. Membrane Sci., 6 (1980) 283.

171 W.D. Munch, L.P. Zestar and J.L. Anderson, Rejection of polyelectrolytes from microporous membranes, J. Membrane Sci.. 5 (1979) 77 .

[8] P.G. de Gennes, Dynamics of entangled polymer solutions. 1. The Rouse model. 2. Inclusion of hydrodynamic interactions, Macromolecules 9 (1976) 587/594

[9] S. Daoudi and F. Brochard, Flows of flexible polymer solutions in pores, Macromolecules, 11 (1978) 751.

$[10]$ Q.T. Nguyen, P. Aptel and J. Néel, Characterization of ultrafiltration membranes. 2. Mass transport measurements for low and high molecular weight synthetic polymers in water solutions, J. Membrane Sci., 7 ( 1980) 141.

[11] Q.T. Nguyen and J. Néel, Characterization of ultrafiltration membranes. 4 . Influence of the deformation of macromolecular solutes on the transport through ultrafiltration membranes, $J$. Membrane Sci., 14 (1983) 111.

[12] T.D. Long and J.L. Anderson, Flow-dependent rejection of polystyrene from microporous membranes, J. Polym. Sci. Polym. Phys. Ed., 22 (1984) 1261. 
| 13| T.D. Long and J.L. Anderson, Effects of solvent goodness and polymer concentration on rejection of polystyrene from small pores, J. Polym. Sci., Polym. Phys. Ed., 23 (1985) 191.

| 14 | S. Poyen, B. Bariou, N. Mameri, M. Portier and M. Bergez, Prediction of rejection coefficients in ultrafiltration, J. Membrane Sci., 43 (1989) 47.

15] B.D. Mitchell and W.M. Deen, Effect of concentration on the rejection coefficients of rigid macromolecules in track-etch membranes, J. Colloid Interface Sci., 113 (1986) 132.

| $16 \mid$ T. Liu, S. Xu, D. Zhang, S. Sourirajan and T. Matsuura, Pore size and pore size distribution on the surface of polyethersulfone hollow fiber membranes, Desalination, 85 (1991) 1 .

| 17| T.D. Long, D.L. Jacobs and J.L. Anderson, Configurational effects on membrane rejection, J. Membrane Sci., 9 (1981) 13.

[18| L. Zeman and M. Wales, Steric rejection of polymeric solutes by membranes with uniform pore size distributions, Sep. Sci. Technol.. 16 (1981) 275

119] C.A. Finch (Ed.). Chemistry and Technology of Water-soluble Polymers, Plenum, New York, 1983.

120| S.W. Shalaby, C.L. McCormick and G.B. Butler (Eds.), Water-soluble polymers, ACS Symp. Ser., 467 (1991).

$1211 \mathrm{~W}$. Norde, Adsorption of proteins from solution at the solidliquid interface, Adv. Colloid Interface Sci., 25 ( 1986) 267.

|22| M.A.M. Beerlage, Polyimide ultrafiltration membranes for non-aqueous systems, Ph.D. Thesis, University of Twente, 1994, Ch. 4, to be published.

|23| F.P. Cuperus, D. Bargeman and C.A. Smolders, A new method to determine the skin thickness of asymmetric UF-membranes using colloidal gold particles, J. Colloid Interface Sci., 135 ( 1990) 486.

|24| K.-J. Kim. V. Chen and A.G. Fane, Ultrafiltration of colloidal silver particles: flux, retention and fouling, J. Colloid Interface Sci., 155 ( 1993) 347.

125| M. Daoud, J.P. Cotton, B. Farnoux, G. Jannink, G. Sarma, H. Benoit. R. Duplessix, C. Picot and P.G. de Gennes, Solutions of flexible polymers. Experiments and interpretation, Macromolecules, 8 ( 1975 ) 804.

|26| R. Nobrega, H. de Balmann, P. Aimar and V. Sanchez, Transfer of dextran through ultrafiltration membranes: a study of rejection data analysed by GPC, J. Membrane Sci., 45 (1989) 17-36.

[27| R.P. Adamski and J.L. Anderson, Configurational effects on polystyrene rejection from microporous membranes, J. Polym. Sci., Polym. Phys. Ed., 25 (1987) 765.

|28| H. de Balmann and R. Nobrega, The deformation of dextran molecules. Causes and consequences in ultrafiltration, $J$. Membrane Sci., 40 ( 1989 ) 311.
[29] P.G. de Gennes, Scaling Concepts in Polymer Physics, Cornell University Press, Ithaca, New York, 1979

[30] P.G. de Gennes, Coil-stretch transition of dilute flexible polymers under ultrahigh velocity gradients, J. Chem. Phys. 60 (1974) 5030.

[31] R.G. Larson and J.J. Magda, Coil-stretch transitions in mixed shear and extensional flows of dilute polymer solutions, Polym. Prepr., 30 (1989) 93.

[32] M.J. Menasveta and D.A. Hoagland, Molecular weight dependence of the critical strain rate for flexible polymer solutions in elongational flow, Macromolecules, 25 (1992) 7060.

|33| T.Q. Nguyen and H.H. Kausch, Chain extension and degradation in convergent flow, Polymer, 33 (1992) 2611.

| 34 | K.A. Narh, J.A. Odell and A. Keller, Temperature dependence of the conformational relaxation time of polymer molecules in elongational flow: invariance of the molecular weight exponent, J. Polym. Sci., Polym. Phys. Ed., 30 ( 1992) 335.

[35] A. Link and J. Springer, Light scattering from dilute polymer solutions in shear flow, Macromolecules, 26 (1993) 464.

[ 36] G. Guillot, L. Léger and F. Rondelez, Diffusion of large flexible polymer chains through model porous membranes, Macromolecules, 18 (1985) 2531.

[37] J. Brandrup and E.H. Immergut, Polymer Handbook, 3d ed.. Wiley, New York, 1989.

|38| I.A. Kathawalla and J.L. Anderson, Pore size effects on diffusion of polystyrene in dilute solution, Ind. Eng. Chem Res., 27 (1988) 866.

[39] M. Doi and S.F. Edwards, The Theory of Polymer Dynamics, Clarendon Press, Oxford, UK, 1986.

| 40 | J. des Cloizeaux and G. Jannink, Polymers in Solution: Their Modeling and Structure, Clarendon Press, Oxford, UK, 1990.

[41] M.T. Bishop, K.H. Langley and F.E. Karasz, Dynamic lightscattering studies of polymer diffusion in porous materials linear polystyrene in porous glass, Macromolecules, 22 (1989) 1220.

[42] I. Teraoka, K.H. Langley and F.E. Karasz, Diffusion of polystyrene in controlled pore glasses: transition from the dilute to the semi-dilute regime, Macromolecules, 26 (1993) 287.

[43] J.A. Creighton, C.G. Blatchford and M.G. Albrecht, Plasma resonance enhancement of Raman scattering by pyridine adsorbed on silver or gold sol particles of size comparable to the excitation wavelength, J. Chem. Soc., Faraday Trans. 2, 75 (1979) 790.

[44] M.A.M. Beerlage, Polyimide ultrafiltration membranes for non-aqueous systems, Ph.D. Thesis, University of Twente, 1994, Ch. 6. to be published. 